\title{
Harmine suppresses the proliferation and migration of human ovarian cancer cells through inhibiting ERK/CREB pathway
}

\author{
JUN GAO $^{1,2,3}$, HONG ZHU $^{3}$, HONG WAN $^{3}$, XIA ZOU $^{3}$, XIAOXIN MA $^{1 *}$ and GUOLAN GAO ${ }^{2 *}$ \\ ${ }^{1}$ Department of Obstetrics and Gynecology, Shengjing Hospital of China Medical University, \\ Shenyang, Liaoning; ${ }^{2}$ Department of Obstetrics and Gynecology, Aviation General Hospital \\ of China Medical University, Beijing; ${ }^{3}$ Department of Obstetrics and Gynecology, \\ The Third Affiliated Hospital of Nanchang University, Nanchang, Jiangxi, P.R. China
}

Received March 21, 2017; Accepted August 3, 2017

DOI: $10.3892 / o r .2017 .5952$

\begin{abstract}
Ovarian cancer is the most lethal gynaecological cancer and the sixth most common cause of cancer related death among Western women. Recent studies show that harmine, a small-molecular $\beta$-carboline alkaloid present in medicinal plants, displayed obvious anticancer effects in several cancer cells. However, the effect of harmine on ovarian cancer is not well understood. In the present study, the effect of harmine on the cell proliferation and migration of ovarian cancer SKOV-3 cells and the underlying mechanism were investigated. Our results indicated that harmine significantly suppressed the proliferation of SKOV-3 cells in a dose-dependent manner. Interestingly, it also inhibited the epidermal growth factor (EGF)-induced proliferation of SKOV-3 cells. Moreover, the migration of SKOV-3 cells was markedly inhibited by harmine treatment. Further study showed that harmine inhibited not only the basal phosphorylation level of extracellular signal-regulated kinase $1 / 2\left(E K_{1 / 2}\right)$ and cyclic adenosine monophosphate response element-binding protein (CREB) but also EGF-induced $\mathrm{ERK}_{1 / 2}$ and CREB phosphorylation. Finally,
\end{abstract}

Correspondence to: Dr Xiaoxin Ma, Department of Obstetrics and Gynecology, Shengjing Hospital of China Medical University, 36 Sanhao Street, Shenyang, Liaoning 110004, P.R. China

E-mail: maxiaoxin666@aliyun.com

Dr Guolan Gao, Department of Obstetrics and Gynecology, Aviation General Hospital of China Medical University, Beijing 100012, P.R. China

E-mail: guolan_gao@163.com

${ }^{*}$ Contributed equally

Abbreviations: CREB, cyclic adenosine monophosphate response element-binding protein; ECM, extracellular matrix; EGF, epidermal growth factor; EGFR, epithelial growth factor receptor; $\mathrm{ERK}_{1 / 2}$, extracellular signal regulated kinase $1 / 2$; MAPK, mitogen activated protein kinase; MMP, matrix metalloproteinase; VEGF, vascular endothelial growth factor

Key words: ovarian cancer, harmine, proliferation, migration harmine significantly suppressed the expression of vascular endothelial growth factor $(V E G F)$ and matrix metalloproteinase (MMP) family $M M P-2$, and $M M P-9$. In conclusion, our data revealed that harmine inhibited the proliferation and migration of SKOV-3 cells, which might be mediated by ERK/CREB pathway. These findings elucidate that harmine may act as a potential therapeutic drug for ovarian cancer treatment.

\section{Introduction}

Ovarian cancer is the sixth most common cause of cancer related death among Western women. Although it represents $30 \%$ of cancers of the female genital tract, ovarian cancers are responsible for more than half of the deaths (1). Significant progress has been made in surgery and the improvement of chemotherapeutic agents in ovarian cancer which led to improved 5-year survival rate, however, ovarian cancer remains the leading cause of death of all gynecological cancers $(1,2)$. The main challenge of current chemotherapies for ovarian cancer treatment is due to drug resistance or relapse. Thus, identification of novel anticancer drugs and understanding the underlying molecular mechanisms will be of great help in the treatment of ovarian cancer.

Natural products have attracted considerable attention due to their potent anticancer effects in the past decades $(3,4)$. It is estimated that over $46 \%$ of the new drugs and new drug candidates for cancer therapy approved by US Food and Drug Administration (FDA) between 1989 and 1995 were natural products or natural products derivatives (5). Notably, recent studies have demonstrated that the $\beta$-carboline alkaloids, a large group of natural and synthetic indole alkaloids that extensively exist in extracts from the leaves, barks, roots and seeds of a variety of plants, possess a broad range of psychopharmacological and neuropharmacological effects (6). Many alkaloids have been widely used in the clinical treatment of cough, hypertension and inflammation. Among them, harmine (7-methoxy-1-methyl-9Hpyrido [3,4-b] indole), which was originally isolated from the seeds of Peganum harmala and Banisteriopsis caapi and has been traditionally used as folk medicine in the Middle East, Central Asia, and South America (7), appears to exhibit various pharmacological effects both in vitro and in vivo, such as anti-Alzheimer, 
anti-inflammatory anxiolytic and antidepressant effects (8). Notably, recent studies also indicate that harmine shows to have significant anticancer effect in several cancer cells including hepatoblastoma HepG2 cells (9), B16F-10 melanoma cells (10) and gastric cancer MGC-803 cells (11). However, the effect of harmine on the proliferation and migration of ovarian cancer cells remains unknown.

To this end, the present study was aimed to investigate the anticancer effect and the possible mechanisms of harminemediated cell proliferation and migration in human SKOV-3 cells. Our results demonstrated that harmine significantly suppressed the proliferation and migration of ovarian cancer cells via inhibition of the ERK/CREB pathway. Moreover, the reduced expression of vascular endothelial growth factor (VEGF), matrix metalloproteinase (MMP)-2 and MMP-9 may be involved in harmine-mediated SKOV-3 cell proliferation.

\section{Materials and methods}

Antibodies and reagents. Antibodies to phospho-ERK $\mathrm{K}_{1 / 2}$ (Thr202/Tyr204) (cat. no. 9101), $\mathrm{ERK}_{1 / 2}$ (cat. no. 9102), phospho-CREB (Ser133) (antibodies to phospho-ERK $1 / 2$ (Thr202/Tyr204) (cat. no. 9198), CREB (antibodies to phospho-ERK ${ }_{1 / 2}$ (Thr202/Tyr204) (cat. no. 9197), $\beta$-actin (antibodies to phospho-ERK $\mathrm{K}_{1 / 2}$ (Thr202/Tyr204) (cat. no. 4970), goat anti-rabbit horseradish peroxidase (HRP)-linked antibody (antibodies to phospho-ERK I/2 (Thr202/Tyr204) (cat. no. 7074) as well as U0126 (specific $\mathrm{ERK}_{1 / 2}$ inhibitor) were purchased from Cell Signaling Technology (Beverly, MA, USA). Harmine, epidermal growth factors (EGF), dimethyl sulfoxide (DMSO) and 3-(4,5-dimethylthiazol-2-yl)2,5-diphenyl tetrazolium bromide (MTT) were purchased from Sigma-Aldrich (St. Louis, MO, USA). Culture medium and other solutions used for cell culture were purchased from Invitrogen (Shanghai, China). SYBR ${ }^{\circledR}$ Premix Ex Taq ${ }^{\mathrm{TM}}$ II was obtained from Takara Bio, Inc. (Otsu, Japan).

Cell culture and treatment. Human SKOV-3 ovarian cancer cells were grown in RPMI-1640 medium supplemented with $10 \%$ fetal bovine serum, $50 \mu \mathrm{g} / \mathrm{ml}$ streptomycin, $50 \mathrm{IU} / \mathrm{ml}$ penicillin. Cultures were maintained at $37^{\circ} \mathrm{C}$ in a humidified atmosphere containing $5 \% \mathrm{CO}_{2}$. Cells were regenerated every 3 days when they reached $70-90 \%$ confluence. Harmine was dissolved in DMSO and diluted to appropriate concentrations with culture medium. The final concentration of DMSO in the culture medium did not exceed $0.1 \%$.

Reverse transcription PCR. Total RNA was extracted using TRIzol reagent. Reverse transcription was performed according to the manufacturer's instructions from Invitrogen. PCR analysis was performed using the following sense and antisense primers: The $V E G F$ primers were forward 5'-CTG GGC TGT TCT CGC TTC G-3' and reverse 5'-CTC TCC TCT TCC TTC TCT TCT TCC-3'. The $M M P-2$ primers were forward 5'-CCG TCG CCC ATC ATC AAG TTC-3' and reverse 5'-GCA GCC ATA GAA GGT GTT CAG G-3'. The $M M P-9$ primers were forward 5'-TGG TCC TGG TGC TCC TGG TG-3' and reverse 5'-GCT GCC TGT CGG TGA GAT TGG-3'. The GAPDH primers were forward 5'-ACA ACT TTG GTA TCG TGG AAG G-3' and reverse 5'-GCC ATC
ACG CCA CAG TTT C-3'. The amplification steps were as follows: $94^{\circ} \mathrm{C}$ for $5 \mathrm{~min}, 30$ cycles of denaturation for $45 \mathrm{sec}$ at $95^{\circ} \mathrm{C}, 45 \mathrm{sec}$ of annealing at $60^{\circ} \mathrm{C}$, elongation at $72^{\circ} \mathrm{C}$ for $45 \mathrm{sec}$, and extension at $72^{\circ} \mathrm{C}$ for $1 \mathrm{~min}$. After amplification, the PCR products were electrophoresed on a $1.5 \%$ agarose gel and visualized by ethidium bromide staining.

Quantitative real-time PCR ( $q R T-P C R)$. cDNA was obtain as described above. PCR primers used for the analysis are listed above. The $20 \mu \mathrm{l}$ PCR system was composed of $2 \mu \mathrm{l} \mathrm{cDNA}$, $10 \mu 1$ SYBR Premix Ex Taq II, $0.4 \mu 1$ ROX Reference Dye II, $0.8 \mu 110 \mu \mathrm{M}$ forward primer, $0.8 \mu 110 \mu \mathrm{M}$ reverse primer

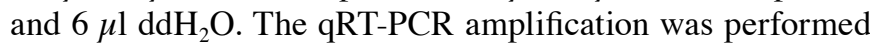
under the following conditions: 1 cycle of initial denaturation for $30 \mathrm{sec}$ at $95^{\circ} \mathrm{C}, 40$ cycles of denaturation for $5 \mathrm{sec}$ at $95^{\circ} \mathrm{C}$, followed by DNA synthesis for $30 \mathrm{sec}$ at $60^{\circ} \mathrm{C}$. After amplification, real-time data acquisition and analysis were performed.

Western blot analysis. After treated with different compounds, cells were harvested at indicated time, washed, and lysed in ice-cold lysis buffer. The cell lysates were sonicated and centrifuged at $12,000 \mathrm{xg}$ at $4^{\circ} \mathrm{C}$ for $5 \mathrm{~min}$. The protein concentration of extracts was determined by using the Bradford reagent from Bio-Rad Laboratories. Equal amounts of protein $(20 \mu \mathrm{g} / \mathrm{lane})$ were resolved with sodium dodecyl sulphate polyacrylamide gel electrophoresis (SDS-PAGE) and transferred to nitrocellulose membranes (Millipore, Bedford, MA, USA). Next, the membrane was blocked in 5\% non-fat dry milk in Tris-buffered saline with Tween-20 for $2 \mathrm{~h}$ at room temperature and then incubated with different primary antibodies overnight at $4^{\circ} \mathrm{C}$. HRP-conjugated secondary antibodies $(1: 20,000)$ were used subsequently for $2 \mathrm{~h}$ at room temperature. Immunoreactive bands were visualized by enhanced chemiluminescence (Pierce, Rockford, IL, USA) and quantified by using ImageJ software (National Institutes of Health, Bethesda, MD, USA).

MTT assay. Cell viability was assessed by MTT assay. Briefly, cells were seeded in a 48 -well flat-bottomed plate $\left(3 \times 10^{4}\right.$ cells per well) and cultured overnight. Then, the cells were starved overnight and treated with different drugs as indicated later. For dose course experiment, the cells were treated with concentrations of harmine and incubated $48 \mathrm{~h}$. For time course experiment, the cells were incubated with $10 \mu \mathrm{M}$ harmine for $12,24,36$, and $48 \mathrm{~h}$. After incubation, $20 \mu \mathrm{l}$ MTT ( $5 \mathrm{mg} / \mathrm{ml})$ was added to each well, and the cells were allowed to grow in complete media at $37^{\circ} \mathrm{C}$ for $3 \mathrm{~h}$. The supernatant was removed, then $500 \mu 1$ DMSO was added to each well and swung for $10 \mathrm{~min}$ to dissolve the crystal. Subsequently, and absorbance was determined by using a microplate spectrophotometer assay reader at $570 \mathrm{~nm}$.

Wound healing assay. SKOV-3 cells were seeded in 6-well plate and cultured to a confluent monolayer. The cells were pretreated with hydroxyurea $(5 \mathrm{mmol} / \mathrm{l})$ to prevent cell proliferation. A pipette tip $(200 \mu \mathrm{l})$ was used to scratch a wound on the midline of each well and the cells were then washed twice with PBS. Following 0, 12, 24, 36 h of culture in RPMI-1640 supplemented with $10 \%$ serum (control) or harmine stimulants, the migration of the cells was evaluated by measuring 

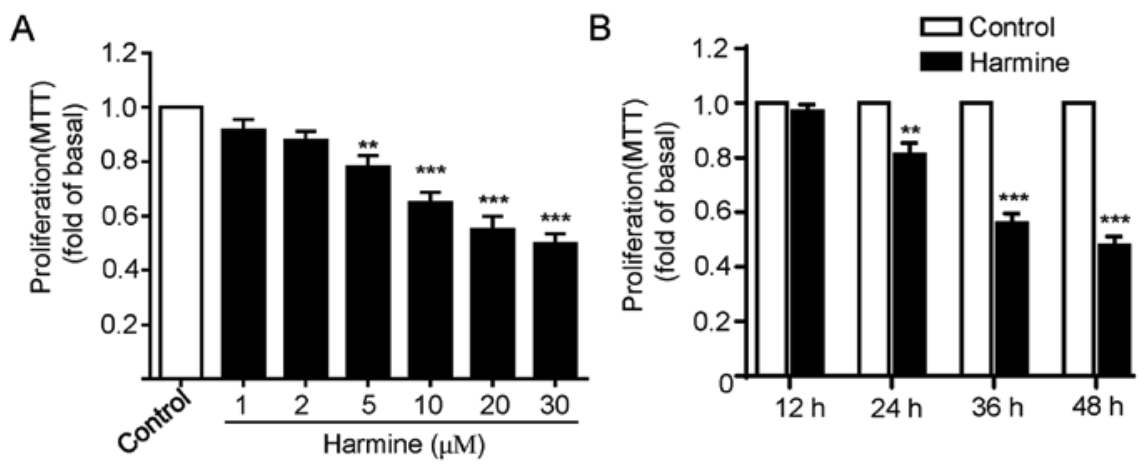

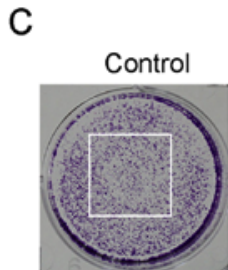

$10 \mu \mathrm{M}$

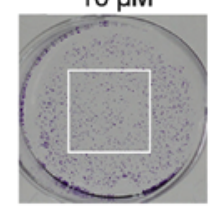

E

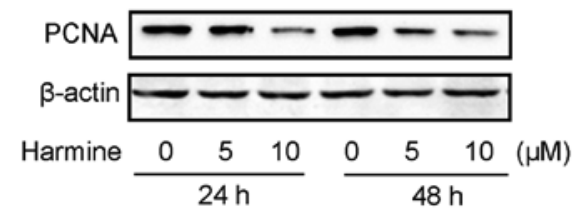

Harmine

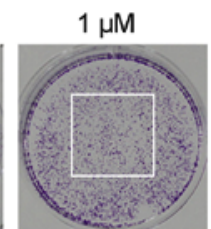

$20 \mu \mathrm{M}$

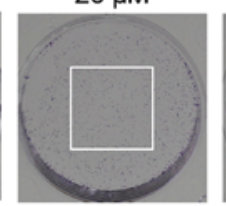

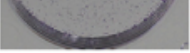

$\mathrm{E}$
$\mathrm{F}$

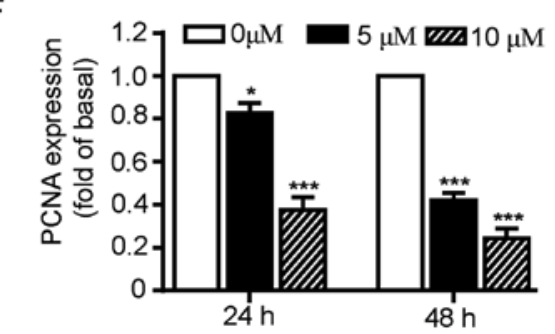

Figure 1. Harmine inhibits the basal cell proliferation level in SKOV-3 cells. (A) Dose-dependent effects of harmine on the proliferation of SKOV-3 cells. Cells were treated with increasing concentrations of harmine as indicated above for $48 \mathrm{~h}$. (B) Time-dependent effects of harmine on the proliferation of SKOV-3 cells. Cells were treated with harmine $(10 \mu \mathrm{M})$ for $12,24,36$ and $48 \mathrm{~h}$. MTT assay was performed to measure the cell viability. Data are representative of triplicate samples from at least three independent experiments and are expressed as the mean \pm SEM. (C) Effects of harmine on colony formation capacity of SKOV-3 cells. Cells were treated with harmine at the indicated concentrations for two weeks. The colony number in the area outlined in white was calculated. (D) Quantitation of colony formation results as shown in (C). (E) The expression of PCNA in SKOV-3 cells was measured by western blot analysis after harmine treatment. (F) Quantitation of western blotting results as shown in (E). ${ }^{*} \mathrm{p}<0.05,{ }^{* *} \mathrm{p}<0.01,{ }^{* * * *} \mathrm{p}<0.001$ vs. control group.

the difference in the area of the wounds with a Leica DM2500 image analysis system (Leica, Mannheim, Germany).

Colony formation assay. SKOV-3 cells in the logarithmic growth phase were seeded in a 6-well plate and cultured in RPMI-1640 medium supplemented with 10\% FBS overnight. Then, the cells were treated with the indicated concentrations of harmine for another two weeks. Then, the supernatants were discarded, and the cells were carefully washed three times with phosphate-buffered saline (PBS). Subsequently, cells were fixed with a fixative composed of methanol-glacial acetic acid (3:1) for $10 \mathrm{~min}$. Finally, the cells were stained in crystal violet for $20 \mathrm{~min}$. Images of the colonies were captured by a digital camera.

Statistical analysis. Data are indicated as mean \pm SEM from at least 3 independent experiments. Statistical analysis was performed using the Student's t-test (comparison of two groups) or one-way analysis of variance (ANOVA) followed by the Dunnett's or Tukey's test (comparison of more than two groups). A value of $\mathrm{p}<0.05$ was considered statistically different. All statistical analyses were conducted using Prism version 6.0 (GraphPad Software).

\section{Results}

Harmine inhibits the basal cell proliferation level in SKOV-3 cells. We first investigated the influence of harmine on the proliferation of SKOV-3 cells. The SKOV-3 cells were incubated with various concentration of harmine $(0,1,2,5,10,20$ and $30 \mu \mathrm{M}$ ) for $48 \mathrm{~h}$ and then cell viability was measured by MTT assay. As shown in Fig. 1A, harmine markedly inhibited the growth of SKOV-3 cells in a dose-dependent manner as concentration of less than $2 \mu \mathrm{M}$ had little effect while concentrations of more than $5 \mu \mathrm{M}$ progressively inhibited the growth of SKOV-3 cells (Fig. 1A). We next explored the effects of harmine at different treatment times on SKOV-3 cell viability, the cells were incubated with $10 \mu \mathrm{M}$ harmine for $12,24,36$ or 

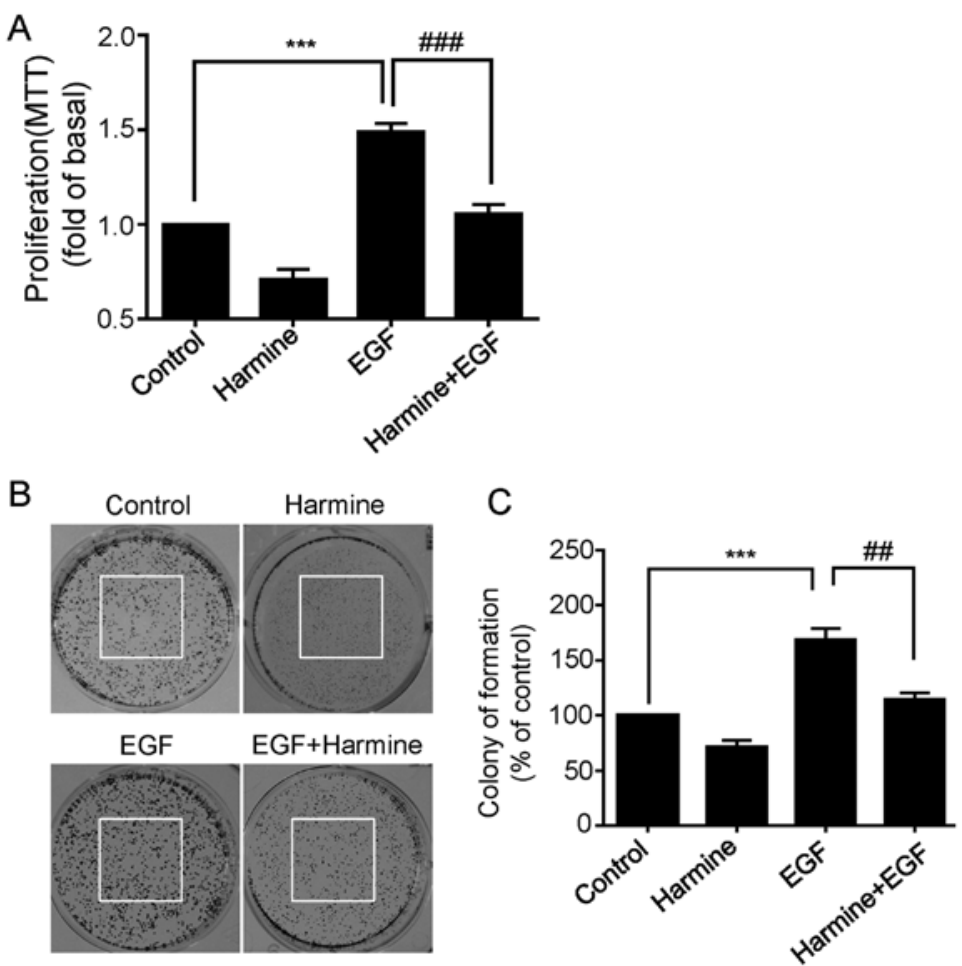

Figure 2. Harmine inhibits EGF-induced SKOV-3 cell proliferation. (A) The cells were treated with harmine (10 $\mu \mathrm{M})$ in the absence or presence of EGF $(50 \mathrm{ng} / \mathrm{ml})$ for $48 \mathrm{~h}$ and then cell viability was measured by MTT assay. (B) Effects of harmine on EGF-induced colony formation capacity of SKOV-3 cells. The colony number in the area outlined in white was calculated. (C) Quantitation of colony formation results as shown in (B). Data presented are mean \pm SEM from at least three independent experiments. ${ }^{* * *} \mathrm{p}<0.001$ vs. control group; ${ }^{\# \#} \mathrm{p}<0.01,{ }^{\# \# \#} \mathrm{p}<0.001$ vs. EGF-treated group.

48 h. Cell viability assays revealed that harmine significantly inhibited SKOV-3 cell growth except $12 \mathrm{~h}$ incubation, with the strongest effect at $48 \mathrm{~h}$ (Fig. 1B).

To further confirm the inhibitory effect of cell proliferation of harmine, colony formation assays were performed. As shown in Fig. 1C, consistent with the MTT assay, harmine significantly suppressed the colony formation of SKOV-3 cells in a dose-dependent manner (Fig. 1C and D). To provide additional evidence, we then detected the expression of proliferating cell nuclear antigen (PCNA), a protein marker that has been widely used for indicating cell proliferation. We found that the expression of PCNA was significantly reduced by harmine treatment for either 24 or $48 \mathrm{~h}$ (Fig. 1E and F). Taken together, our above results strongly indicated that harmine showed significant inhibitory effect toward the cell growth in SKOV-3 cells.

Harmine inhibits EGF-induced proliferation of SKOV-3 cells. It is widely known that epidermal growth factor receptor (EGFR) is often overexpressed in numerous human cancers including ovarian cancer, and activation of EGFR is known to be involved in the growth and progression of a variety of malignancies $(12,13)$. We next asked whether harmine was able to inhibit EGF-induced cell proliferation of SKOV-3 cells. To do this, the cells were treated with harmine in the absence or presence of EGF $(50 \mathrm{ng} / \mathrm{ml})$ for $48 \mathrm{~h}$ and then cell viability was measured by MTT assay. As shown in Fig. 2A, EGF significantly increased the proliferation of SKOV-3 cells, while this EGF-enhanced proliferation was remarkably suppressed by harmine treatment (Fig. 2A). Consistent with the previously results, harmine not only inhibited EGF-enhanced proliferation, but also inhibited
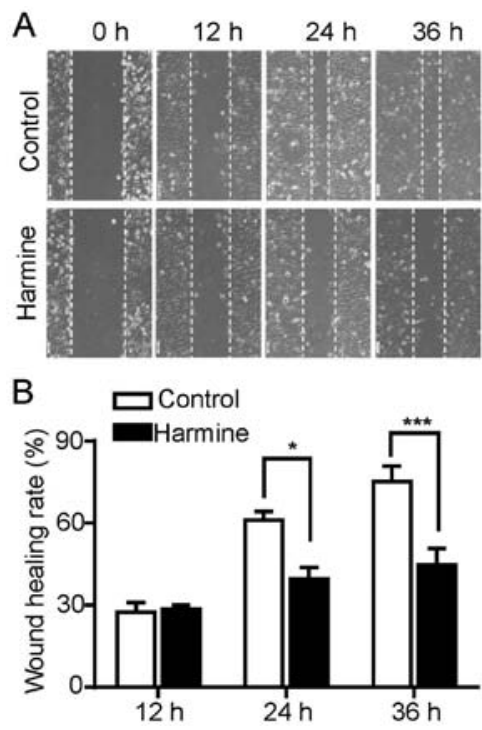

Figure 3. Harmine significantly suppresses the migration of SKOV-3 cells (A) Cells were treated with hydroxyurea $(5 \mathrm{mmol} / \mathrm{l})$ to prevent proliferation then effect of harmine on the migration of SKOV-3 cells was measured by wound healing assay. (B) Relative closed-wound distance was calculated by measuring the width of at least four wounds and normalized to control. Data presented are mean \pm SEM. ${ }^{*} \mathrm{p}<0.05,{ }^{* * * *} \mathrm{p}<0.001$ vs. control group.

basal proliferation level of the SKOV-3 cells in the absence of EGF (Fig. 2A). Similar results were observed by colony formation assay. As expected, harmine significantly inhibited EGF-induced colony formation capacity (Fig. 2B and C). These results further confirmed that harmine could obviously inhibit ovarian cancer cell proliferation. 


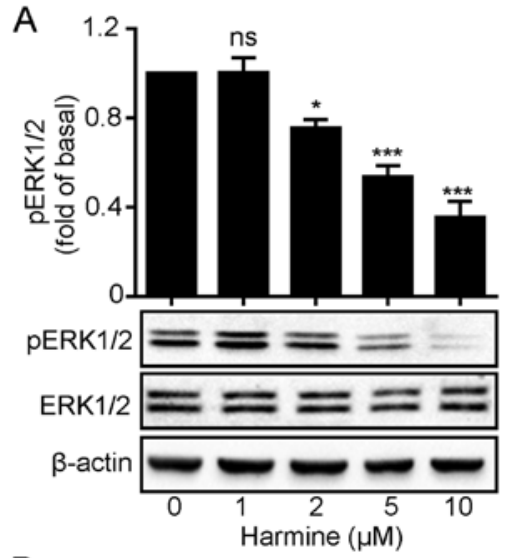

B

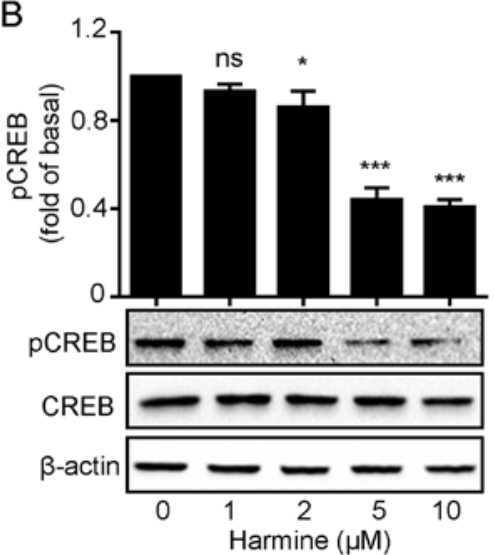

Figure 4. Harmine inhibits $\mathrm{ERK}_{1 / 2}$ and CREB phosphorylation in SKOV-3 cells. Upper panel, dose course of the $\mathrm{ERK}_{1 / 2}$ (A) and CREB (B) phosphorylation after incubation with various concentration of harmine. Lower panel, typical immunoblots used to quantify the phosphorylated $E_{R K} K_{1 / 2}\left(\mathrm{pERK}_{1 / 2}\right)$ (A) and CREB (B). Data represent the mean \pm SEM from at least four independent experiments. ${ }^{*} \mathrm{p}<0.05,{ }^{* * * *} \mathrm{p}<0.001 \mathrm{vs}$. control group.

Harmine significantly suppresses the migration of SKOV-3 cells. Cell migration plays an important role during cancer progression. To explore the effect of harmine on the migration of SKOV-3 cells, a typical scratch wound healing assay was performed to measure the migration ratio. As our above results indicated that harmine was able to inhibit the proliferation of SKOV-3 cells, this may interfere with the analysis of cell migration results. Thus, to exclude the proliferation inhibitory effect of harmine on migration, the wound healing assay were performed in the presence of hydroxyurea which prevents cell proliferation by inhibiting the DNA synthesis. As shown in Fig. 3, harmine significantly inhibited the migration of SKOV-3 cells at 24 and $36 \mathrm{~h}$ after treatment while had little effect at $12 \mathrm{~h}$ (Fig. 3A and B).

Harmine blocks ERK $K_{1 / 2}$ and CREB phosphorylation in SKOV-3 cells. We demonstrated that harmine significantly suppressed SKOV-3 cell growth. This raised the question how harmine acts to inhibit SKOV-3 cell growth. Mitogen-activated protein kinase (MAPK) pathways constitute a large modular network that regulates a variety of physiological processes including cell growth and differentiation. Thus, we firstly monitored $\mathrm{ERK}_{1 / 2}$ phosphorylation levels following harmine treatment. As shown in Fig. 4, harmine caused a rapid decrease in the phosphorylation level of $\mathrm{ERK}_{1 / 2}$ with no changes in total
ERK $_{1 / 2}$ expression levels (Fig. 4A). Similarly, the phosphorylation level of CREB, an important transcription factor that plays important roles in cell proliferation, was also decreased in the same manner as $\mathrm{ERK}_{1 / 2}$ (Fig. 4B), which might suggest that harmine inhibited $\mathrm{ERK}_{1 / 2}$ phosphorylation which in turn suppressed CREB activation.

Harmine inhibits EGF-induced $E R K_{1 / 2}$ and CREB phosphorylation in SKOV-3 cells. Harmine significantly inhibited the cell proliferation and $\mathrm{ERK}_{1 / 2}$ and CREB phosphorylation in SKOV-3 cells, however, we do not know whether the inhibitory effect of harmine on cell proliferation was mediated by ERK $_{1 / 2} / \mathrm{CREB}$ pathway. To verify this, we next investigated whether harmine could block EGF-induced ERK $_{1 / 2}$ and CREB phosphorylation. As shown in Fig. 5, we first confirmed that EGF treatment significantly increased the phosphorylation of ERK $_{1 / 2}$ and CREB (Fig. 5A and B). However, both EGF-induced ERK $_{1 / 2}$ and CREB phosphorylation were remarkably inhibited by harmine treatment (Fig. 5A and B). To demonstrate that CREB phosphorylation was downstream of $\mathrm{ERK}_{1 / 2}$, we evaluated the effect of the $\mathrm{EKR}_{1 / 2}$ selective inhibitor on EGF-induced CREB phosphorylation in SKOV-3 cells. Cells were pretreated for 30 min with U0126 $(10 \mu \mathrm{M})$ and then stimulated with EGF. We found that U0126 blocked EGF-induced ERK 1/2 $_{2}$ and CREB (Fig. 5C and D) phosphorylation, suggesting CREB acted as a downstream factor of $\mathrm{ERK}_{1 / 2}$.

Harmine reduces the expression of VEGF, MMP-2 and $M M P-9$ in SKOV-3 cells. Since vascular endothelial growth factor (VEGF) and matrix metalloproteinases (MMPs), particularly the gelatinases MMP-2 and MMP-9, have been shown to play important roles in the proliferation, migration and metastasis of many primary epithelial tumors $(14,15)$, we then examined the effect of harmine on the expression of VEGF, MMP-2 and MMP-9 in SKOV-3 cells. As shown in Fig. 6A, reverse transcription-PCR analysis showed that the gene transcription of VEGF, MMP-2 and $M M P-9$ were significant downregulated in the presence of harmine $(10 \mu \mathrm{M})$ for $48 \mathrm{~h}$ (Fig. 6A and B). Consistently, quantitative real-time PCR analysis also indicated that harmine markedly suppressed the mRNA level of $V E G F$ (Fig. 6C), MMP-2 (Fig. 6D) and MMP-9 (Fig. 6E).

\section{Discussion}

In the present study, we investigated the antitumor effect and potential mechanism of harmine on human ovarian cancer cells. Our results showed that harmine not only significantly inhibited the proliferation and migration of SKOV-3 cells, but also suppressed the EGF-induced proliferation of SKOV-3 cells. Moreover, this antitumor effect of harmine might be through inhibiting the $\mathrm{EKR}_{1 / 2} / \mathrm{CREB}$ pathway and downregulation the expression of VEGF and MMPs.

Many natural alkaloids such as camptothecin, vincristine and ellipticine are found to be potent anticancer agents in recent years (16). These alkaloids kill tumor cells by different biochemical modes of action, such as inhibition of apoptosis, inhibition of topoisomerase I and II and inhibition of microtubule formation. However, the molecular mechanisms for the antitumor activity of $\beta$-carbolines are not fully defined. Of 

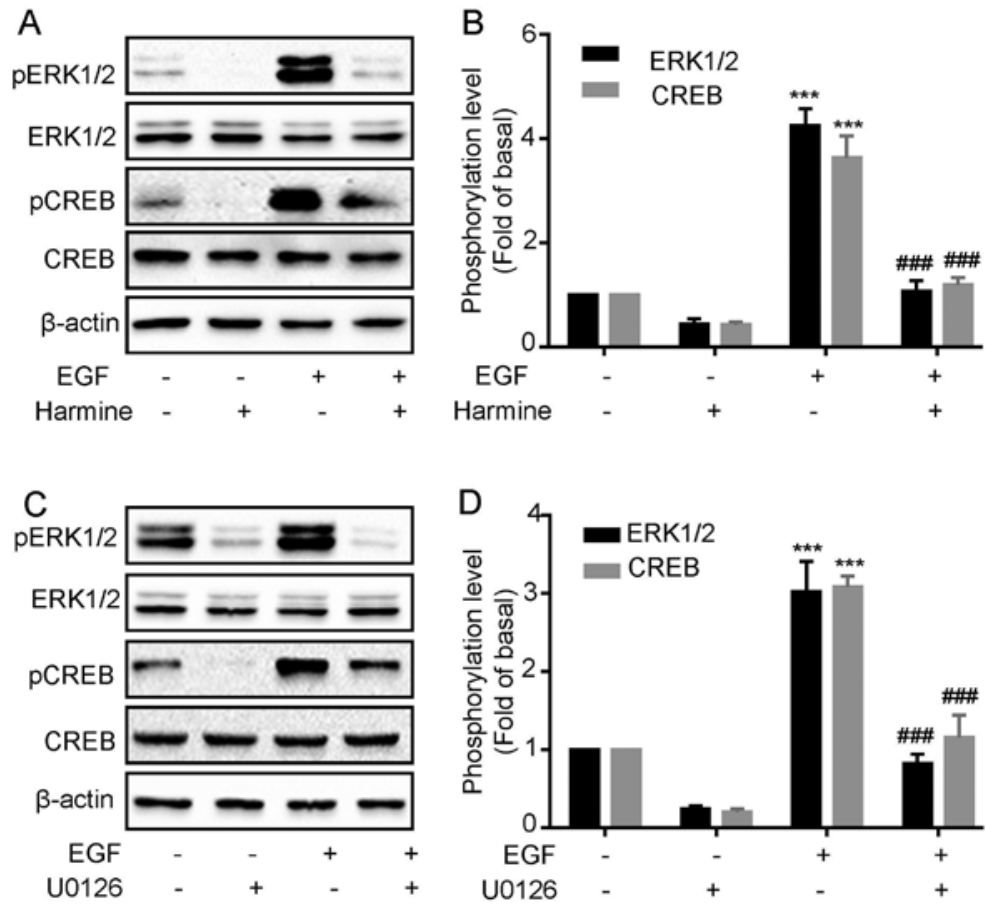

Figure 5. Harmine inhibits EGF-induced ERK $1 / 2$ and CREB phosphorylation in SKOV-3 cells. (A) Effects of harmine on EGF-induced ERK ${ }_{1 / 2}$ and CREB phosphorylation. (B) The relative densities of protein bands shown in (A) were analyzed and normalized to $\beta$-actin. (C) Effects of MEK M $_{1 / 2}$ inhibitor U0126 on EGF-induced ERK $_{1 / 2}$ phosphorylation. (D) The relative densities of protein bands shown in (C) were analyzed and normalized to $\beta$-actin. Data represent the mean \pm SEM from at least three independent experiments. ${ }^{* * *} \mathrm{p}<0.001$ vs. control group, ${ }^{\# \# \#} \mathrm{p}<0.001$ vs. EGF-treated group.

A

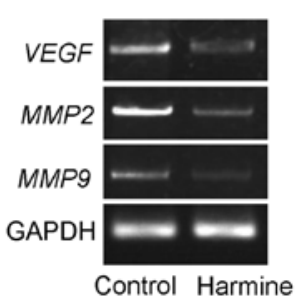

B

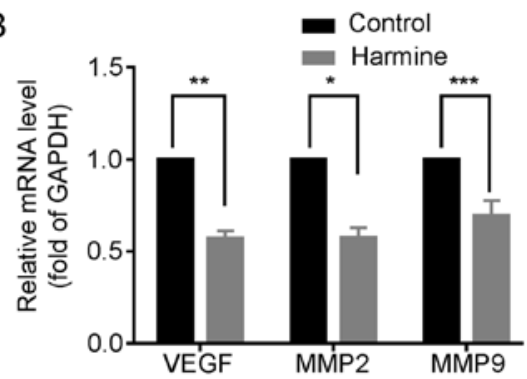

$\mathrm{E}$
C

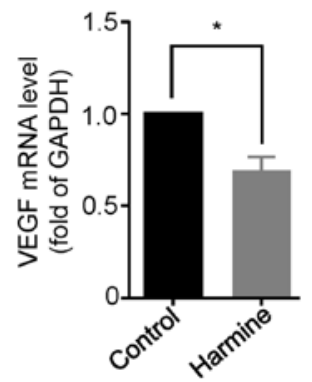

D

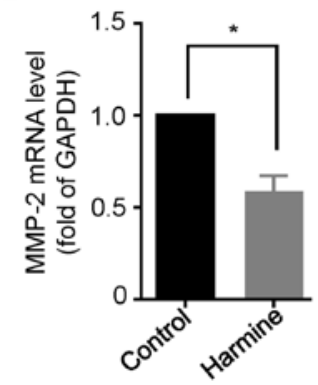

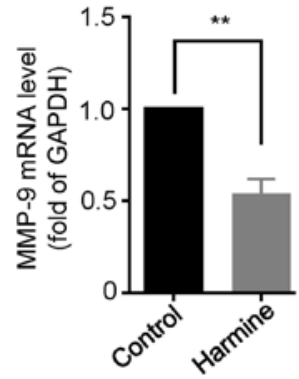

Figure 6. Harmine reduces the expression of $V E G F, M M P-2$ and $M M P-9$ in SKOV-3 cells. (A) Cells were treated with $10 \mu \mathrm{M}$ harmine for $48 \mathrm{~h}$, mRNA level of VEGF, MMP-2 and MMP-9 were detected by reverse transcription PCR. (B) Quantitation of reverse transcription PCR results as shown in (A). RT-qPCR was performed to detect the mRNA level of $V E G F$ (C), MMP-2 (D) and MMP-9 (E). Data are shown as means \pm SEM of three triplicate experiments. " $\mathrm{p}<0.05$, ${ }^{* *} \mathrm{p}<0.01,{ }^{* * *} \mathrm{p}<0.001$ vs. control group.

note, it is reported that some $\beta$-carbolines and their related compounds appear to have cytotoxic effects on several cell lines including cancer cells and cerebellar granule neurons. Though cytotoxic agents which result in cell death and eventually tumor shrinkage are conventional anticancer drugs and still the most widely used around the world (17), the non-cytotoxic, molecularly targeted drugs that inhibit tumor growth without direct cytotoxicity is of greatest concern (18). In our study, the inhibitory effect of cell growth in the presence of harmine may result from two possible reasons, from the unspecific cytotoxic effect of harmine that killed the cancer cells acutely, or that harmine may suppress the proliferation of SKOV-3 cells without inducing acute cellular damage. To discern the later from the former, we measured 
the cell viability of SKOV-3 cells treated with or without $10 \mu \mathrm{M}$ harmine for $12 \mathrm{~h}$. Our results showed that there was no significant difference between the cell viability of control cells and harmine-treated cells. On the other hand, a recent study indicated that treatment with harmine increased proliferation of cultured neural progenitor cells without DNA damage or cell death (19). Moreover, harmine was found to promote cell proliferation as exposure of chick embryos to harmine markedly increased BrdU incorporation and number of mitotic cells in the spinal cord (20). Together with our results, one can speculate that the inhibition of the proliferation of SKOV-3 cells by harmine may not be due to its unspecific cytotoxic effect.

Our results showed that harmine inhibited the basal proliferation level of SKOV-3 cells. Noteworthy, it also significantly suppressed EGF-induced proliferation of SKOV-3 cells, suggesting an ideal antitumor effect of harmine. Aberrant expression and signaling of the EGFR-mediated pathway are associated with various human cancers including breast, colon, head and neck, pancreatic, lung and ovarian cancers. Undoubtedly, EGFR and its family members have emerged as the most useful biomarkers and rational target for cancer therapy (21). It is worth noting that EGFR has been reported to be overexpressed in up to $60 \%$ of ovarian cancers (22), while increased expression and activation of EGFR is related to high cell proliferation index, high migration and stromal invasion rate $(23,24)$. This may explain, to some extent, why harmine inhibits the basal proliferation level of SKOV-3 cells. However, how harmine affect EGFR signaling pathway needs to be further investigated.

Mitogen-activated protein kinase (MAPK) pathways such as $\mathrm{ERK}_{1 / 2}$ play an important role in the regulation of proliferation and migration of cancer cells. Consistent with the results showing harmine inhibited the proliferation of SKOV-3 cells, the phosphorylation of $\mathrm{ERK}_{1 / 2}$ was profoundly blocked by harmine treatment. Moreover, harmine also blocked the CREB phosphorylation which was reported to suppress apoptosis, induce cell proliferation, migration and mediate tumor metastasis (25). Since the inhibition manner of CREB phosphorylation was quite in accordance with $\mathrm{ERK}_{1 / 2}$, suggesting that CREB acts downstream of $\mathrm{ERK}_{1 / 2}$. This hypothesis was confirmed by the following data. First, we observed that the phosphorylation of ERK $\mathrm{K}_{1 / 2}$ and CREB mediated by EGF were markedly inhibited in the presence of harmine. Second, the EGF-induced CREB phosphorylation can be significantly blocked by U0126, an inhibitor widely used to block ERK $\mathrm{ER}_{1 / 2}$ activity, which indicates EGF-induced CREB phosphorylation is mediated by $\mathrm{ERK}_{1 / 2}$. Thus, we conclude that inhibition of MEK/ERK pathway which in turn blocked CREB activity is involved in harmine's suppressive effect on EGF-induced cell proliferation in human ovarian cancer cells.

Degradation of extracellular matrix (ECM) and basement membranes by the tumor cells is a critical step in the processes of tumor invasion and metastasis (26). Matrix metalloproteinases (MMPs) are a family of proteolytic enzymes that regulate various cell behaviors with relevance to cancer biology through degradation of extracellular matrix surrounding tumors (14). In our study, we found that harmine can inhibit the expression of $M M P-2$ and $M M P-9$. Early adhesion and metastasis of ovarian cancer cells are mediated by the MMP family of proteins, the expression of which is upregulated during ovarian cancer progression (27). Though MMPs have been considered to be closely correlated with tumor invasion and metastasis (27), studies also show that inhibition of the activities of $M M P-2$ and $M M P-9$ can result in reduced ECM remodeling and suppression of endothelial cell proliferation and migration (28). Moreover, consistent with our results, a recent study indicates that activation of $\mathrm{ERK}_{1 / 2} / \mathrm{CREB}$ pathway promotes the expression of $M M P-2$ and $M M P-9$ which may account for lung cancer cell proliferation (29). On the other hand, our results also showed that harmine can inhibit the expression of VEGF. Since VEGF is the most important mitogenic and survival factors involved in angiogenesis while angiogenesis is pre-requisite for the growth of solid tumors, vascular targeting has been explored as a potential strategy to suppress tumor growth and metastasis. Thus, this result further confirmed the antitumor effect of harmine. However, whether harmine is able to inhibit angiogenesis in the development of a solid tumor needs further study.

\section{Acknowledgements}

The present study was supported by grants from the Traditional Chinese Medicine Research (no. 2016B032), Science and Technology Planning Project (no. 20175032) of Jiangxi Provincial Health and Family Planning Commission, the Guiding Science and Technology Program of Nanchang (no. 2016-ZDXXM-039).

\section{References}

1. Torre LA, Bray F, Siegel RL, Ferlay J, Lortet-Tieulent J and Jemal A: Global cancer statistics, 2012. CA Cancer J Clin 65: 87-108, 2015.

2. Coleman RL, Monk BJ, Sood AK and Herzog TJ: Latest research and treatment of advanced-stage epithelial ovarian cancer. Nat Rev Clin Oncol 10: 211-224, 2013.

3. Mukherjee AK, Basu S, Sarkar N and Ghosh AC: Advances in cancer therapy with plant based natural products. Curr Med Chem 8: 1467-1486, 2001.

4. McChesney JD: Natural products in drug discovery - organizing for success. P R Health Sci J 21: 91-95, 2002.

5. Cragg GM, Newman DJ and Snader KM: Natural products in drug discovery and development. J Nat Prod 60: 52-60, 1997.

6. Cao R, Peng W, Wang Z and Xu A: beta-Carboline alkaloids: Biochemical and pharmacological functions. Curr Med Chem 14: 479-500, 2007.

7. Patel K, Gadewar M, Tripathi R, Prasad SK and Patel DK: A review on medicinal importance, pharmacological activity and bioanalytical aspects of beta-carboline alkaloid 'Harmine'. Asian Pac J Trop Biomed 2: 660-664, 2012.

8. Farzin D and Mansouri N: Antidepressant-like effect of harmane and other beta-carbolines in the mouse forced swim test. Eur Neuropsychopharmacol 16: 324-328, 2006.

9. Cao MR, Li Q, Liu ZL, Liu HH, Wang W, Liao XL, Pan YL and Jiang JW: Harmine induces apoptosis in HepG2 cells via mitochondrial signaling pathway. Hepatobiliary Pancreat Dis Int 10: 599-604, 2011.

10. Hamsa TP and Kuttan G: Harmine activates intrinsic and extrinsic pathways of apoptosis in B16F-10 melanoma. Chin Med 6: 11, 2011.

11. Zhang P, Huang CR, Wang W, Zhang XK, Chen JJ, Wang JJ, Lin C and Jiang JW: Harmine hydrochloride triggers G2 phase arrest and apoptosis in MGC-803 cells and SMMC-7721 cells by upregulating p21, activating caspase-8/Bid, and downregulating ERK/Bad pathway. Phytother Res 30: 31-40, 2016.

12. Lafky JM, Wilken JA, Baron AT and Maihle NJ: Clinical implications of the ErbB/epidermal growth factor (EGF) receptor family and its ligands in ovarian cancer. Biochim Biophys Acta 1785: 232-265, 2008. 
13. Liu LZ, Hu XW, Xia C, He J, Zhou Q, Shi X, Fang J and Jiang BH: Reactive oxygen species regulate epidermal growth factor-induced vascular endothelial growth factor and hypoxiainducible factor-1alpha expression through activation of AKT and P70S6K1 in human ovarian cancer cells. Free Radic Biol Med 41: 1521-1533, 2006.

14. Newby AC: Matrix metalloproteinases regulate migration, proliferation, and death of vascular smooth muscle cells by degrading matrix and non-matrix substrates. Cardiovasc Res 69: 614-624, 2006.

15. Bernatchez PN, Soker S and Sirois MG: Vascular endothelial growth factor effect on endothelial cell proliferation, migration, and platelet-activating factor synthesis is Flk-1-dependent. J Bio Chem 274: 31047-31054, 1999.

16. Demain AL and Vaishnav P: Natural products for cancer chemotherapy. Microb Biotechnol 4: 687-699, 2011.

17. Kummar S, Gutierrez M, Doroshow JH and Murgo AJ: Drug development in oncology: Classical cytotoxics and molecularly targeted agents. Br J Clin Pharmacol 62: 15-26, 2006.

18. Fox E, Curt GA and Balis FM: Clinical trial design for targetbased therapy. Oncologist 7: 401-409, 2002.

19. Dakic V, Maciel RM, Drummond H, Nascimento JM, Trindade $P$ and Rehen SK: Harmine stimulates proliferation of human neural progenitors. PeerJ 4: e2727, 2016.

20. Hämmerle B, Ulin E, Guimera J, Becker W, Guillemot F and Tejedor FJ: Transient expression of Mnb/Dyrk1a couples cell cycle exit and differentiation of neuronal precursors by inducing p27KIP1 expression and suppressing NOTCH signaling. Development 138: 2543-2554, 2011.
21. Seshacharyulu P, Ponnusamy MP, Haridas D, Jain M, Ganti AK and Batra SK: Targeting the EGFR signaling pathway in cancer therapy. Expert Opin Ther Targets 16: 15-31, 2012.

22. Dimova I, Zaharieva B, Raitcheva S, Dimitrov R, Doganov N and Toncheva D: Tissue microarray analysis of EGFR and erbB2 copy number changes in ovarian tumors. Int J Gynecol Cancer 16: 145-151, 2006.

23. Dancey JE and Freidlin B: Targeting epidermal growth factor receptor - are we missing the mark? Lancet 362: 62-64, 2003

24. Lassus H, Sihto H, Leminen A, Joensuu H, Isola J, Nupponen NN and Butzow R: Gene amplification, mutation, and protein expression of EGFR and mutations of ERBB2 in serous ovarian carcinoma. J Mol Med (Berl) 84: 671-681, 2006.

25. Aggarwal S, Kim SW, Ryu SH, Chung WC and Koo JS: Growth suppression of lung cancer cells by targeting cyclic AMP response element-binding protein. Cancer Res 68:981-988, 2008.

26. Lu P, Weaver VM and Werb Z: The extracellular matrix: A dynamic niche in cancer progression. J Cell Biol 196: 395-406, 2012.

27. Egeblad M and Werb Z: New functions for the matrix metalloproteinases in cancer progression. Nat Rev Cancer 2: 161-174, 2002.

28. Gomez DE, Alonso DF, Yoshiji $\mathrm{H}$ and Thorgeirsson UP: Tissue inhibitors of metalloproteinases: Structure, regulation and biological functions. Eur J Cell Biol 74: 111-122, 1997.

29. Hu P, He J, Liu S, Wang M, Pan B and Zhang W: $\beta 2$-adrenergic receptor activation promotes the proliferation of A549 lung cancer cells via the ERK1/2/CREB pathway. Oncol Rep 36: $1757-1763,2016$ 